\title{
CT and UltraSound in the diagnosis of orbital dermoid cysts - A retrospective study at the University Teaching Hospital in Lusaka
}

\author{
Luan Chun min \\ Radiology Department, \\ University Teaching Hospital, Lusaka, Zambia
}

\begin{abstract}
Objective: To evaluate the use of Computed Tomography (CT) and Ultrasound (US) to diagnose orbital dermoid cyst and compare the characteristic features of CT and US in detection of the lesions.
\end{abstract}

Design: CT and Ultrasound were used to evaluate patients with orbital dermoid cysts in 16 cases were retrospectively analysed.

Result: Ultrasound features were confirmatory of orbital demoid cysts in 4 cases where as CT was effective in diagnosing the condition in 15 cases except for one case of mistaken diagnosis for mucocoele. The characteristic features as fat-floating sign on CT were revealed in 7 patients.

Conclusion: Ultrasound is useful in displaying internal structures, CT is excellent in detecting the orbital demoid cyst and can more accurately confirm the diagnosis when the characteristic features as fat -floating sign is revealed.

\section{INTRODUCTION}

Dermoid cyst accounts for about 3-9\% of all orbital masses $^{1,2}$. Ultrasound and CT are the most import examinative modalities of orbital dermoid cysts. In order to evaluate the use and compare the characteristics of Ultrasound and CT scan in detection of dermoid cyst, 16 cases of this lesion which were proved by operation, and them were taken for the study from Jun, 2003 to Jun, 2008 in University Teaching Hospital (UTH), Lusaka and Sinozam Hospital, Kitwe, Zambia.

Key words: Computed Tomography, Ultrasound, orbital, dermoid cyst.

\section{MATERIAL AND METHODS}

16 cases ( 9 male, 7 female) of orbital demoid cysts from 2-47 years old (average 23 years old) and 9 cases being under 10 years old. Clinically, patients presented with gradually proptosis, ptosis of the eye lids, orbital congestion, ametropia or impairment of vision. Sixteen cases were examined by Ultrasound and CT scan before operation. The final diagnosis were confirmed by operation and histological examination. US were performed with a high resolution, $10 \mathrm{MHz}$ real-time sector probe. CT scan were performed on Philip Aura CT scanner in UTH, Lusaka and IQ/TC Picker scanner in Kitwe, Zambia. With and without contrast CT scan using $2 \mathrm{~mm}$ sections in axial position were taken through bilateral orbits.

\section{RESULTS}

Ultrasound features were confirmatory of orbital Demoid cysts in 4 cases where as CT was effective in diagnosing the condition in 15 cases, except for one case of mistaken diagnosis for mucocoele. In 6 cases were involved to ertra ocular muscles, 9 cases were involved to both ocular muscles and retrobulbar space. Intraorbital mass with intracranial extension is revealed in one patient. In 6 cases showed uniformly anechogenic content which were not to diagnosed as cysts, 9 cases demonstrated complex echogenic masses, in which 4 cases were given defined diagnosis of dermoid cyst, and one patient was not identified on US. Fifteen cases were detected as oval, semicircular well defined (12/16) or ill defined (4/16) masses with densities ranging from that of fat $(-150$ $\mathrm{Hu}$ ) to lower soft tissue (unto $25 \mathrm{Hu}$ ) on CT scan. Seven cases presented with the characteristic features known as the "fat-floating" sign. Capsule calcificated plaques were noted in 5 cases. Orbital walled abnormalities as different degrees of depression or 
defect were detected in 9 cases. Lesions involved both orbits in 2 cases (Fig1, 2). Following contrast CT scan 5 cases showed capsuled or peripheral enhancement.

\section{DISCUSSION}

Dermoid cyst is an ectodermal inclusion cyst which may occur anywhere in the body, but it's occurrence in orbit is relatively rare. Approximately $50 \%$ of tumours that involve the head are found in or adjacent to the orbit. Dermoid cyst is a common benign intraorbital neoplasm, and it is usually cystic ${ }^{3}$. There are a variety of cystic and cyst- like lesions which involve the orbit in paediatric patients. The developmental orbital cysts include choristoma (epidermoid, dermoid, dermolipoma) teratoma and the congenital cystic eye. Most of orbital dermoid cysts are caused by developmental sequestrations of surface epidermis, often adjacent to bony structure lines or sutures. It is often found after a long period of slow growth in adults. However, hidden rupture of orbital dermoid cysts occurred proportionately most often at the age of 20 to 40 which results in perisitent granulomatous inflammations ${ }^{4}$. In case of rupture of a intraorbital demoid cyst with intracranial extension, a granulomatous inflammatory reaction is incited leading to chemical meningitis, seizures, vasospasm with infarction and death ${ }^{5,6}$. Thus Klin et al recommend an early operative removal of demoid cysts, if possible at the age of 3 or 4 , but at least with in the first 10 years ${ }^{5}$.

Pathologically demoid cysts are well defined lobulated masses containing thick viscous, oily fluid and cholesterol derived from decomposed epithelial cells. Dermal appendages like hairs, sebaceous and sweat gland are also seen ${ }^{2,7}$. By way of rupture, the content of the cyst gets into contact with the surrounding tissue, which results in a granulomatous reaction to the foreign body with a pseudo-capsule. Aetiologically a hidden rupture is promoted by the growth of the dermoid cyst and the pubertal enlargement of the sebaceous glands in the wall of cyst ${ }^{4}$.

Imaging in orbital dermoid cyst is valuable in diagnosis characterization of benign lesion and to demonstrate their intraorbital and intracranial extension. The most important of investigative modalities are X-ray, Ultrasound, CT and Magnetic Resonace Imaging (MRI). As Ultrasound does not use $\mathrm{x}$-rays or ionizing radiation, it can be considered as part of the initial office examination and may be repeated without risk to the patient .In this study, US detected complex echogenic masses in 9 patients, in which only 4 cases were given definitive diagnosis of dermoid cysts. Ultrasonography failed to diagnosis as cysts, lesions which appeared as uniform lesion with anechogenic contents in 6 patients. There was also one patient where Ultrasound failed to identify the cyst because the lesion was small and located at the orbital apex region. The orbital wall limited the use of US in detecting some intraorbital lesions; however US has the advantage of displaying the internal structures and the dynamics of orbital dermoid cysts.

CT scan is valuable for the diagnosis of intraorbital dermoid cyst, which have complex components and different densities. Sixteen cases were detected as oval, semi-circular well defined (12/16) or ill defined (4/16) masses with densities ranging from that of fat $(-150 \mathrm{Hu})$ to lower soft tissue $(25 \mathrm{Hu})$ on nonenhanced CT scan, following intravenous contrast administration, there are 5 patients had evidence of enhancement in the capsule or peripheral component. Otherwise the central components did not enhancement of dermoid cysts. These abnormalities could be related to the granulomatous reaction resulting from a ruptured dermoid cyst. In nonruptured cases, the capsular enhancement is not very frequently seen, finding in the study are comparable to those reported by Kaufma ${ }^{9}$. There was one case of mistaken diagnosis for mucocoele from the ethmoid sinus. The reason for this misdiagnosis was the presence of only liquid contained in the lesion. Therefore, orbital demoid cysts could be diagnosed accurately if the fatty components is found within it. In this study, there were characteristic CT features in 7 patients, with appearances of the upper component of the lesion which were a transparent field of fat and the lower component were relatively dense. It is called the "fat-floating" sign (fig. 3) in this study. Most of the orbital dermoid cysts are situated outside the periosteum, they often displace the orbital walls or cause sharply demarcated defect in the orbital bones adjacent to the lesion. In this study, 9 cases had bony changes in orbital walls, and different degrees of digital depression or bony crest formation presented (fig. 4). It is an important sign on CT scan, aids in the diagnosis of orbital demoid cyst. CT scan has the advantage of detecting the orbital wall abnormality which is in contrast to Ultrasound scan. 
The various differential diagnoses of dermoid cyst are epidermoid cyst, arachnoid cyst and various nonneoplastic, non-inflammatory developmental and acquired cysts. Epidermoid cyst cannot be distinguished clinically from dermoid cyst. On enhanced CT scan epidermoid cysts appear as low density (similar to CSF) mass lesion with occasional peripheral enhancement and calcifications. There are not highly uncommon ${ }^{9}$. The uniformed liquid hypodense dermoid cysts poses a diagnostic challenge to radiologist in distinguishing between it with other orbital cystic lesions.

Thus this study shows that Ultrasound is a useful

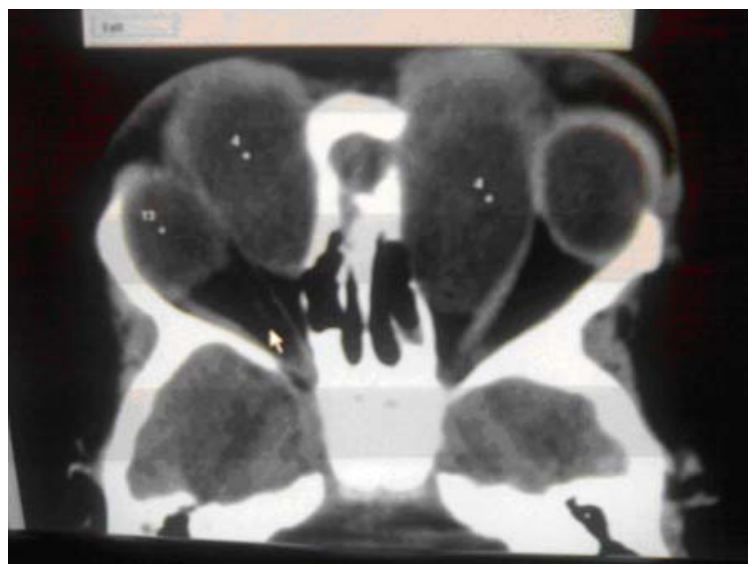

FIG 1. Case 1. A 3-year-old male patient. Axial view show: uniformed hypodense Dermoid cysts involved to bilateral orbits in medial extraocular muscle spaces which displaced both medial recti and eyeballs.

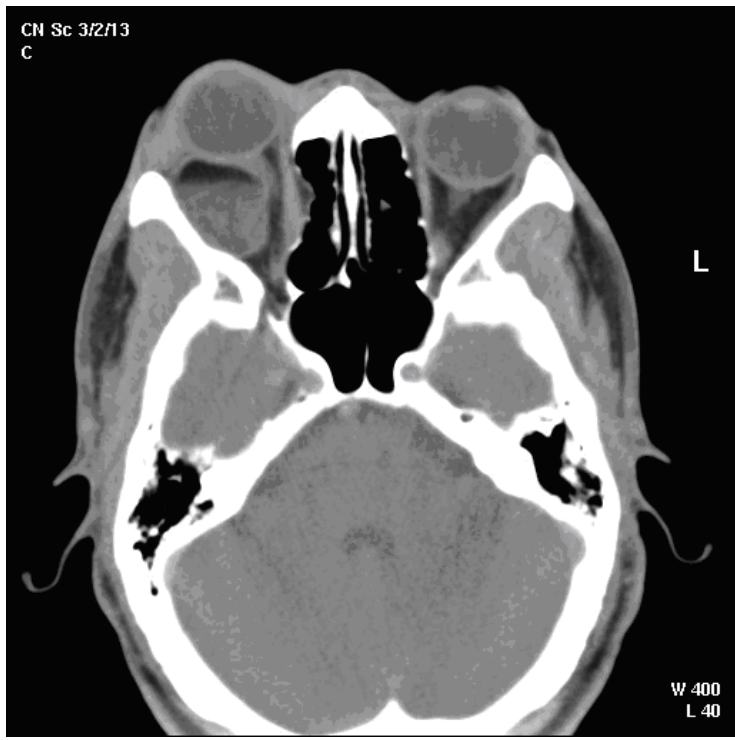

FIG 3. Case 2. A 26-year-old male patient. An axial enhanced CT scan detect: a dermoid cyst involved to left orbit both in exta-occura muscles space and retraocular space. The upper component was transparent field of fat, the lower component was relatively dense of the mass, it is the characteristic feature as "fat-floating" sign of dermoid cyst method and CT is excellent in diagnosing orbital dermoid cysts. The "fat-floating" sign in orbital mass is the characteristic feature in diagnosis of orbital dermoid cysts. An orbital mass containing complex components of different densities from fat to liquid with or without compressive changes are useful for diagnosis of orbital dermoid cyst on CT. An illdefined orbital dermoid cyst with capsular or peripheral enhancement, is highly suggestive of ruptured dermoid cyst. Though CT is superior, Ultrasound being more economical and without radiative risk can be used as initial or follow-up examination once the diagnosis has been made.

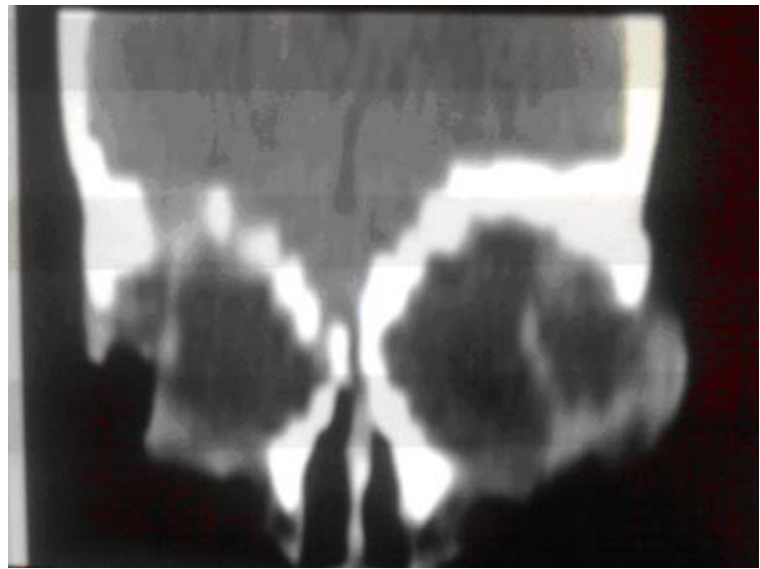

FIG 2. The same patient to fig 1. Bilateral eyeball were displaced laterally by dermoid cysts, on sagittal reconstruction view.

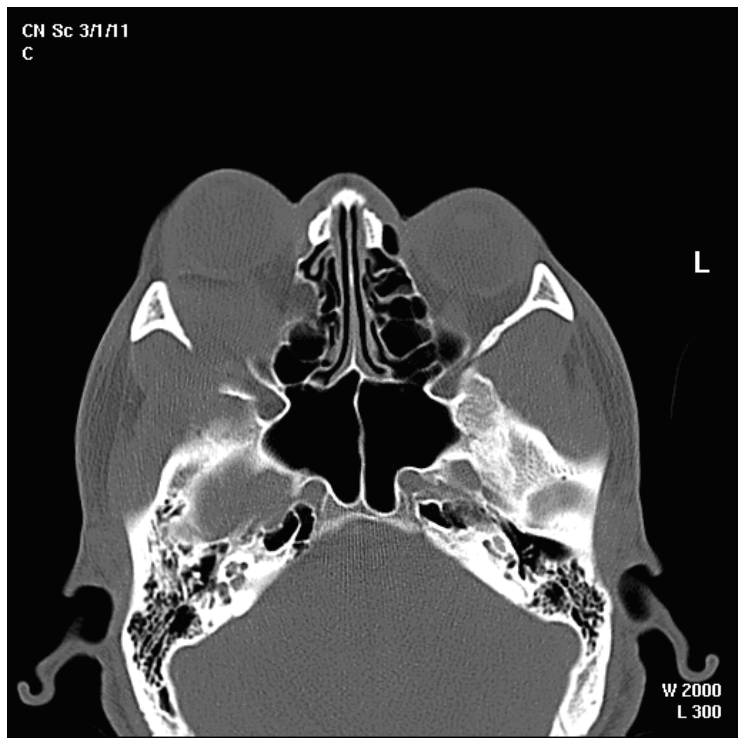

FIG 4. The same patient to fig 3 . bone window show orbital walled defect present in demoid cyst. 


\section{REFERENCES}

1. Pfeiffer R L, Nicholl R J. Dermoid -Epidermoid Tumours of orbit. Arch Ophthalmology 1948; 46:39

2. Osborn A G. Diagnostic Neuroradiology, Mosby, 1997:66-67,631-48

3. Son Guo-xiang. Orbital lesion; Enlightenment from 1012 operation. Asian Hospital 1989; 8:23

4. Klin Monastsble Augenheilkd. Covered rupture of periocular dermoid cysts. Clinico-histologic study 1993; Dec: 203:403-7

5. Sherman R P, Rootman J, Lapoint J S. Dermoid clinical presentation and management. $\mathrm{Br} J$ Ophthalmol 1984; 68:642-52
6. Albert D M, Jakobief F A. Principles and Practice of Ophthalmology. W B Saunder Company, Philadelphia, 2000.Vol. 4: pp 3072-81

7. Handoff M Fine B S. Ocular Pathology, $3^{\text {rd }}$ Ed. Philadelphia: Harper \& Row, 1988; 520.

8. Shi Ji tong, Son Guo xiang, Xiao Li lua, et Al. Photography diagnosis for common intraorbital tumours. Chin J Ophthalmol 1997; 33; 92-5.

9. Kaufman L M, Villablanca J B, Mafee M F. The Radiological clinics of North America.W B Saunders Company, Philadelphia, 1998. Vol. 136, No .6, 1149-63. 